\title{
Primary Glioblastoma of the Cerebellopontine Angle : Case Report and Review of the Literature
}

\author{
Ji-Hye Lee, M.D., Jong Hyun Kim, M.D., Ph.D., ${ }^{1}$ Taek-Hyun Kwon, M.D., Ph.D. ${ }^{2}$ \\ Department of Neurosurgery, VHS Medical Center, Seoul, Korea \\ Department of Neurosurgery, ${ }^{2}$ Korea University Guro Hospital, Korea University College of Medicine, Seoul, Korea
}

Glioblastoma multiforme (GBM) is located most frequently in the cerebral hemispheres. Glioblastoma presenting as an extraaxial mass of cerebellopontine angle (CPA) is very rare in adults. We report a rare case of GBM arising in the CPA. The patient was a 71-year-old female, who complained of progressive gait disturbance and poor memory. Initial magnetic resonance imaging (MRI) revealed a $1.4 \times 1.3 \mathrm{~cm}$ mass in the left CPA, with broad base to the petrous bone, showing homogenous enhancement. Follow-up MRI showed a rapid increase in size of mass $(2.7 \times 2.2 \mathrm{~cm})$ with a necrotic portion. A stereotactic biopsy was done under the guidance of navigation system, and the histopathologic diagnosis was GBM, World Heath Organization grade IV. Further surgical resection was not performed considering her general condition, and the patient underwent concurrent chemotherapy with radiation therapy. Although rare, the possibility of glioblastoma should be included in the differential diagnosis of atypical CPA tumor.

Key Words : Glioblastoma multiforme · Cerebellopontine angle · Extraaxial.

\section{INTRODUCTION}

Glioblastoma multiforme (GBM) is the most common primary brain tumor in adults, comprising about $20 \%$ of all intracranial tumors ${ }^{8,10)}$. GBM usually arises in the supratentorial region, most commonly cerebral hemispheres ${ }^{8}$. Primary infratentorial GBM is an uncommon disease in adults and rarely found in cerebellopontine angle (CPA), especially ${ }^{8,10)}$. A few cases of GBM arising in CPA have been reported in the literature ${ }^{1,2,5-7,9,11)}$. Most of cases reported are primary intraaxial GBM arising from cerebellar hemisphere or brainstem, with exophytic extension into CPA. One case of primary extraaxial GBM in the CPA has been reported, arising from the region of root entry zone of the eighth cranial nerve ${ }^{10)}$.

This case report describes the rare case of a GBM arising in the CPA, completely separated from the brainstem, in a 71-year-old female patient.

\section{CASE REPORT}

A 71-year-old woman was admitted complaining of progressive gait disturbance and poor memory for 3 months. No remarkable disease or trauma history was noted. A neurological examination revealed a disturbance of tandem gait and difficulty in balancing. There was no other neurological sign

- Received : March 9, 2015 • Revised : May 6, 2015 •Accepted : June 23, 2015

- Address for reprints : Taek-Hyun Kwon, M.D., Ph.D.

Department of Neurosurgery, Korea University Guro Hospital, Korea University College of Medicine, 148 Gurodong-ro, Guro-gu, Seoul 08308, Korea Tel : +82-2-2626-3097, Fax : +82-2-863-1684, E-mail : ns806@gmail.com

This is an Open Access article distributed under the terms of the Creative Commons Attribution Non-Commercial License (http://creativecommons.org/licenses/by-nc/4.0) which permits unrestricted non-commercial use, distribution, and reproduction in any medium, provided the original work is properly cited. 
such as motor weakness, cranial nerve deficit, ataxia, or long tract sign. A laboratory examinations and plain radiographic images of skull and cervical spine were unremarkable.

Initial magnetic resonance imaging (MRI) demonstrated a well-defined $1.4 \times 1.3 \mathrm{~cm}$ lobulated mass in the left CPA, with broad base to the petrous bone. The mass was adherent to cerebellum and completely separated from the brainstem, suggesting extraaxial tumor (Fig. 1). T2-weighted MRI showed abnormal high signal intensity in cerebellum and brainstem around the mass. The mass was enhanced homogenously on a gadolinium enhanced T1-weighted image. In suspicion of common extraaxial tumor in the CPA such as meningioma, follow-up image was taken after 3 months. Follow-up MRI showed a rapid increase in size of mass $(2.7 \times 2.2 \mathrm{~cm})$, which
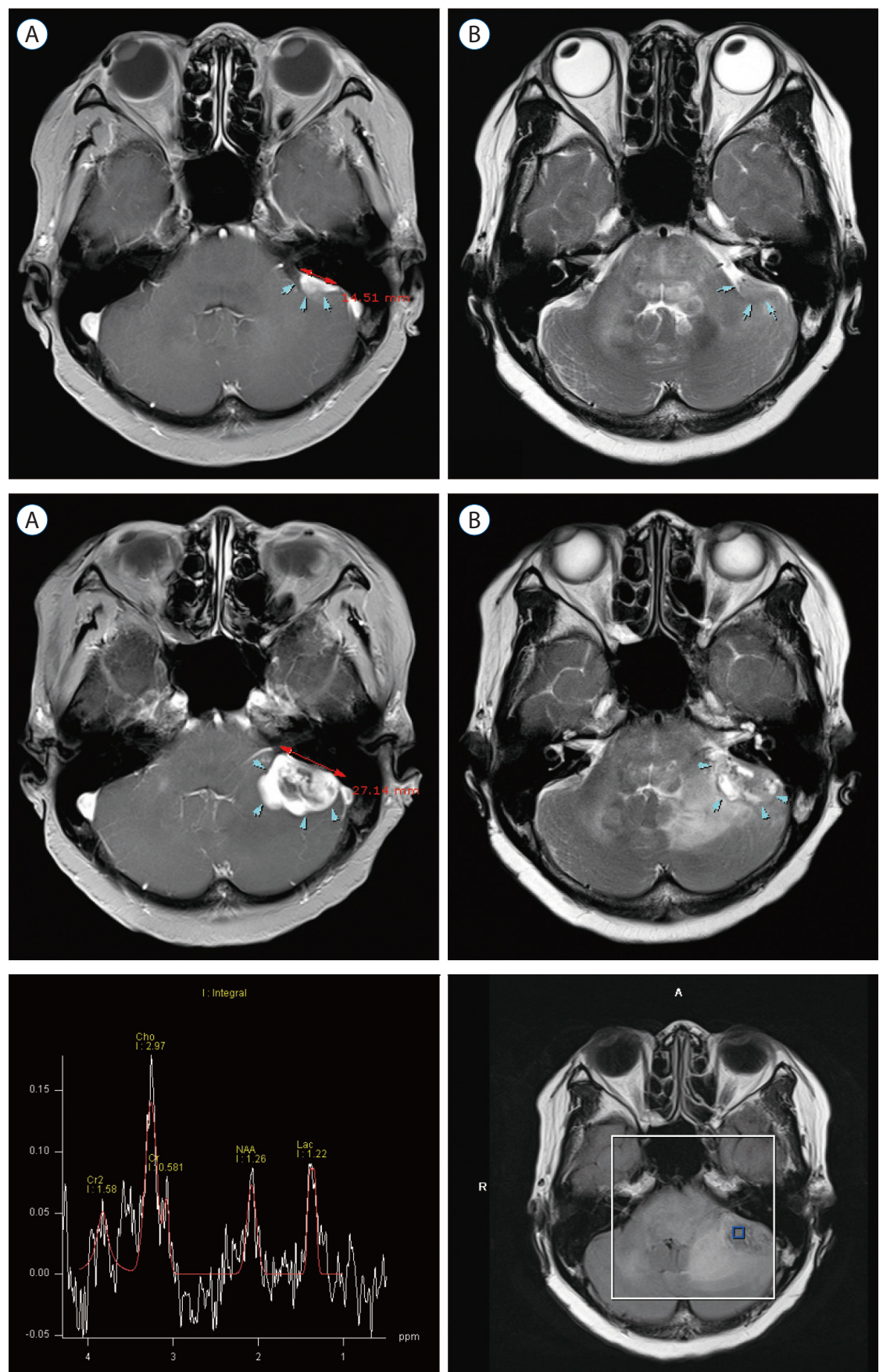

Fig. 1. Initial magnetic resonance imaging scans with axial enhanced T1-weighted (A) and T2weighted (B) images, showing the mass in the left cere- bellopontine angle with broad base to the petrous bone (arrows).

Fig. 2. 3 months-follow-up magnetic resonance imaging scans with axial (A) T1-weighted and T2weighted (B) images reveal rapid increase in size of mass and extensive peritumoral edema (arrows).

Fig. 3. Magnetic resonance spectroscopy demonstrates an increased choline to creatine ratio, decreased $\mathrm{N}$-acetyl aspartate, and increased lactate. This findings indicates the possibility of high grade tumor. 

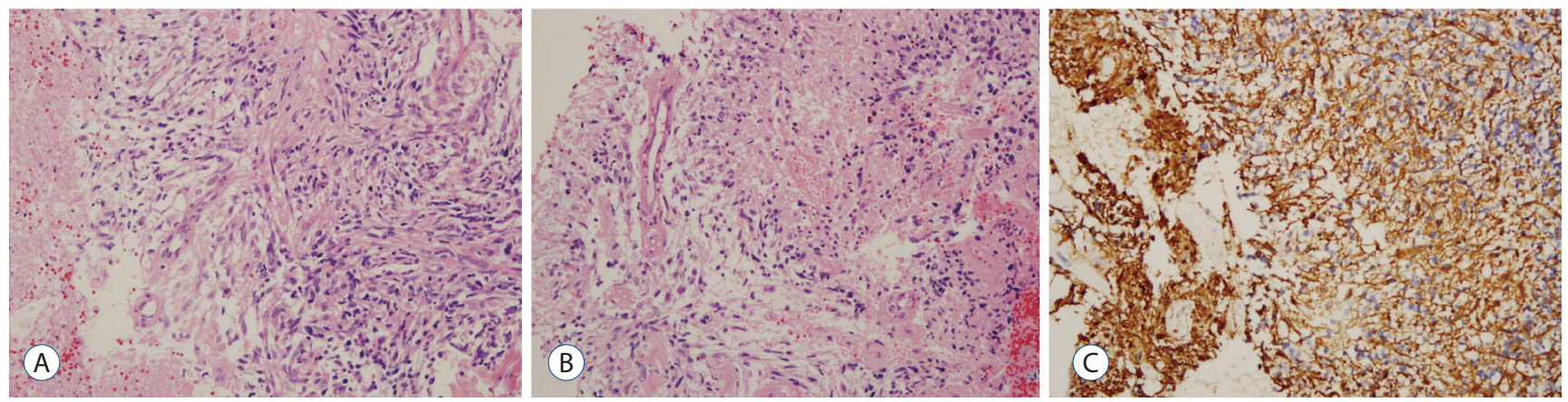

Fig. 4. A : Histopathology of the tumor shows atypical glial cells with frequent mitotic activity and moderate nuclear atypia (hematoxylin and eosin [H\&E], 200x). B : Marked microvascular proliferation in the tumor (H\&E, 200X). C : Immunohistochemical staining for GFAP reveals diffuse immunoreactivity in the tumor cells (200x). GFAP : glial fibrillary acidic protein.

Table 1. Case reports of primary glioblastomas in the cerebellopontine angle

\begin{tabular}{|c|c|c|c|c|c|}
\hline Authors, year & Sex/Age & Origin & Symptoms duration & Treatment & Prognosis \\
\hline $\begin{array}{l}\text { Swaroop and Whittle, } \\
1997\end{array}$ & $M / 22$ & Pons & 1 year & Subtotal resection & Unknown \\
\hline Kasliwal et al., 2008 & $M / 11$ & Cerebellum & 15 days & Subtotal resection & Dead after 2 months \\
\hline Rasalingam et al., 2008 & $M / 9$ & Pons & 2 weeks & Subtotal resection & Alive after 2 months \\
\hline Wu et al., 2011 & $M / 60$ & CN VIII & 2 months & Subtotal resection & Dead after 2 months \\
\hline Salunke et al.," 2012 & $M / 59$ & Pons & 3 months & $\begin{array}{l}\text { Subtotal resection, } \\
\text { and radiation therapy }\end{array}$ & Unknown \\
\hline Matsuda et al., 2013 & $M / 69$ & Cerebellum & 1 hour & $\begin{array}{l}\text { Subtotal resection, and concurrent } \\
\text { radiation therapy with } \\
\text { chemotherapy (temozolomide) }\end{array}$ & Alive after 24 months \\
\hline
\end{tabular}

CN : cranial nerve.

was enhanced peripherally with central necrotic portion on a gadolinium enhanced T1-weighted image (Fig. 2). For differential diagnosis of the mass, magnetic resonance spectroscopy (MRS) was taken, and it demonstrated an increased choline to creatine ratio, decreased $\mathrm{N}$-acetyl aspartate, and increased lactate, considering the possibility of metastatic tumor, high grade glioma, lymphoma, or less likely atypical meningioma (Fig. 3).

To confirm the diagnosis, the patient underwent a stereotactic biopsy under the guidance of navigation system. Entry point was $4 \mathrm{~cm}$ lateral to inion and $2 \mathrm{~cm}$ inferior to transverse sinus, and multiple gray-whitish, gelatinous tissues were obtained. There was no complicated event during biopsy.

Histopathologic examination revealed atypical glial cells with frequent mitotic activity and moderate nuclear atypia. Marked coagulation necrosis and microvascular proliferation were present diffusely within the tumor (Fig. 4). Pseudopali- sading necrosis was not observed. Immunohistochemical staining of the tumor cells revealed diffuse glial fibrillary acidic protein activity, and the proliferation rate was 30\% determined by Ki-67 labeling index. The tumor cells were negative for CD3, CD10, CD20, MUM-1 and BCL-6. These findings were consistent with a GBM, WHO grade IV.

The postoperative course was uneventful. Considering the general condition and age, the patient was not to undergo further surgical removal of the tumor. Conventional radiotherapy was performed, concurrent with temozolomide therapy. The patient has remained symptom free for 1 year since the diagnosis.

\section{DISCUSSION}

Primary CPA glioblastoma is a rare entity in adults ${ }^{8,10)}$. Most 
of the gliomas including GBM occur in the cerebral hemispheres, and posterior fossa glioma is uncommon, estimating as $1.5 \%$ in the cerebellum and $4.1 \%$ in the brainstem ${ }^{3}$. However primary GBM arising in the CPA is further rare with a few reported cases ${ }^{1,2,5-7,9-11)}$. To our knowledge, six cases have been reported in the literatures ${ }^{2,5-7,9,10)}$. Table 1 summarizes the cases of primary GBMs in the CPA.

Primary CPA glioblastoma can be categorized into two types depending on the origin of the tumor. The first category of the CPA glioblastoma is an intraaxial tumor originated from brainstem or cerebellum with an exophytic growth into the $\mathrm{CPA}^{4-7,9,11)}$. This type of GBM is rare, and especially cerebellar GBM with the exophytic growth pattern is very rare, and up to date, four cases have been reported in the literature ${ }^{2,4,5,11)}$. Two cases showed exophytic growth into CPA, and the other two cases were in the crural/quadrigeminal cistern and cisterna magna, respectively.

The second category is an extraaxial CPA glioblastoma. There has been one case of primary extraaxial GBM in the $\mathrm{CPA}$, arising from the proximal portion of cranial nerve $\mathrm{VIII}^{10)}$. Some possible mechanisms were documented regarding the origin of primary extraaxial GBM in the $\mathrm{CPA}^{1,10)}$. One is that the tumor arose from cranial nerve system tissue within proximal cranial nerve itself, and the other is that the tumor originated from heterotopic neuroglial cells in the leptomeninges covering the proximal cranial nerve or brainstem ${ }^{1,10)}$. In our case, the tumor was completely separated from the brainstem and adherent to the cerebellum, suggesting the possibility of an intraaxial GBM originating in the cerebellum or an extraaxial CPA glioblastoma.

A problem among these CPA glioblastomas including the present case is the difficulty of preoperative diagnosis. These cases could be misdiagnosed as extraaxial benign tumors presented more commonly. In our case, initial MRI findings suggested an extraaxial benign tumor such as a CPA meningioma. However, the aggressive clinical course, such as short duration of symptoms and rapid growth pattern of the mass, did not favor such a benign lesion. In addition, MRS was performed for the differentiation of malignancy from benign tumors, and it showed the findings of malignant tumors.

Considering poor general condition and age of the patient in the present case, stereotactic biopsy was chosen as the first plan of management. Histopathologic examination of the present case revealed typical features of GBM. Immunohisto- chemical examination for the differentiation of lymphoma was also performed, but all were negative for lymphomaCD3, CD10, CD20, MUM-1, and BCL-6.

The current standard treatment for GBM includes a maximum resection of the tumor, followed by concurrent chemotherapy (temozolomide) and radiation therapy ${ }^{8,10)}$. However the optimal management for the CPA glioblastoma is to be defined because of its rarity. In the present case, surgical resection was not performed in consideration of the poor general condition, and radiation therapy with concurrent temozolomide therapy was applied.

\section{CONCLUSION}

We report a rare case of primary glioblastoma arising in the CPA. Although this is a rare occurrence, GBM should be considered in the differential diagnosis of an atypical lesion of the cerebellopontine angle in adults.

\section{References}

1. Arnautovic KI, Husain MM, Linskey ME : Cranial nerve root entry zone primary cerebellopontine angle gliomas : a rare and poorly recognized subset of extraparenchymal tumors. J Neurooncol 49 : 205-212, 2000

2. Kasliwal MK, Gupta DK, Mahapatra AK, Sharma MC : Multicentric cerebellopontine angle glioblastoma multiforme. Pediatr Neurosurg 44 : 224-228, 2008

3. Larjavaara S, Mäntylä R, Salminen T, Haapasalo H, Raitanen J, Jääskeläinen J, et al. : Incidence of gliomas by anatomic location. Neuro Oncol 9 : 319-325, 2007

4. Linsenmann T, Monoranu CM, Westermaier T, Varallyay C, Ernestus RI, Vince GH : Exophytic glioblastoma arising from the cerebellum : case report and critical review of the literature. J Neurol Surg A Cent Eur Neurosurg 74 : 262-264, 2013

5. Matsuda M, Onuma K, Satomi K, Nakai K, Yamamoto T, Matsumura A : Exophytic cerebellar glioblastoma in the cerebellopontine angle : case report and review of the literature. J Neurol Surg Rep 75 : e67-e72, 2014

6. Rasalingam K, Abdullah JM, Idris Z, Pal HK, Wahab N, Omar E, et al. : A rare case of paediatric pontine glioblastoma presenting as a cerebellopontine angle otogenic abscess. Malays J Med Sci 15 : 44-48, 2008

7. Salunke P, Sura S, Tewari MK, Gupta K, Khandelwal NK : An exophytic brain stem glioblastoma in an elderly presenting as a cerebellopontine angle syndrome. Br J Neurosurg 26 : 96-98, 2012

8. Stark AM, Maslehaty H, Hugo HH, Mahvash M, Mehdorn HM : Glioblastoma of the cerebellum and brainstem. J Clin Neurosci 17 : 12481251, 2010 
9. Swaroop GR, Whittle IR : Exophytic pontine glioblastoma mimicking acoustic neuroma. J Neurosurg Sci 41 : 409-411, 1997

10. Wu B, Liu W, Zhu H, Feng H, Liu J : Primary glioblastoma of the cerebellopontine angle in adults. J Neurosurg 114 : 1288-1293, 2011
11. Yamamoto M, Fukushima T, Sakamoto S, Tsugu H, Nagasaka S, Hirakawa $\mathrm{K}$, et al. : Cerebellar gliomas with exophytic growth--three case reports. Neurol Med Chir (Tokyo) 37 : 411-415, 1997 